\title{
Wireless Communication-Based Design and Implementation of Digital Protection Platform on Folk Art
}

\author{
Longyu Gong \\ College of Art, Hunan City University, Yiyang 413000, Hunan, China \\ Correspondence should be addressed to Longyu Gong; gonglongyu@hncu.edu.cn
}

Received 16 December 2021; Revised 13 January 2022; Accepted 20 January 2022; Published 11 February 2022

Academic Editor: Deepak Kumar Jain

Copyright (c) 2022 Longyu Gong. This is an open access article distributed under the Creative Commons Attribution License, which permits unrestricted use, distribution, and reproduction in any medium, provided the original work is properly cited.

\begin{abstract}
Numbers no longer only have a purely mathematical meaning but a code that can change the way of human existence, cognition, and thinking, and a basic element to convey information. With the advent of the "Internet+" era, the development of digital media technology, digitization, and informatization has been integrated into all walks of life and has a vital impact on the development of all walks of life, and also brought about the ideological change of fine arts. Nowadays, the digitization of fine arts is a new product of the computer age, and how to protect it is also an important topic. The continuous development of wireless mobile communications has promoted the rapid development of microprocessors. In this article, we will study the protective effect of microprocessors on the digitization of fine arts. This paper designs a microprocessor-based art digital protection website, with the purpose of preserving, inheriting, and disseminating folk art works through the website. The result of the experiment shows that the sampling rate of the microprocessor has reached the requirement of the signal. The largest number of users of the folk art digital website is young people aged 18-29, accounting for 32\%; the highest average score for the website is those over 59 years old, with an average score of 90.
\end{abstract}

\section{Introduction}

1.1. Background. As one of the ways of information transmission, traditional physical display can no longer meet people's needs. The display with the help of digital technology has become a trend in the dissemination of information in various industries in the society; especially in the display and dissemination of cultural heritage in recent years, a large number of digital display methods have been used. For example, cultural heritages such as sculpture works, drama works, and folk music works are kept in digital form. In the fine art industry, by changing the style and function, folk art can be turned into a modern art work, the essence of folk culture can be extracted, the inheritance of specific elements of folk art can be completed, and the folk art culture can be inherited. The rapid development of processors has made the research of microprocessors a hot spot in the field of research at home and abroad. As the most important part of embedded systems, it is widely used in many fields, and it is of no exception in the field of digital art protection.
1.2. Significance. The continuous progress of human civilization has promoted the development of technology and means of realization. At the same time, the progress of technical means has in turn provided more possibilities for the presentation of human civilization. Using the latest digital information technology, intervening in the management of cultural heritage resources, converting cultural heritage into digital form, and establishing a cultural heritage protection mechanism adapted to the digital age are of great significance to the protection of cultural heritage. Keeping up with the development and progress of digital media technology, using digital means to assist the protection, inheritance, and development of folk arts and crafts is gradually becoming a new field and a new focus in new media development. It is believed that the application of microprocessors will bring new vitality to the digital protection of fine arts.

1.3. Related Work. In the protection of folk art works, the digitization of fine arts has become unstoppable. So far, many scholars have conducted research on the digitization 
of fine arts. Burns discussed the importance of photographic documents and the inherent characteristics of digital objects, and discussed the feasibility of digitization as a method of archival photography preservation. By exploring the concepts of originality and aura, Burns proposed and deconstructed a popular view that the digital version of the material photos retains the reproduction of the object content rather than the reproduction of its material form [1]. Rushmeier et al. focused on the application of arts and cultural heritage. For decades, graphics have played a role in the digitization, restoration, protection, display, and dissemination of cultural heritage, and have provided a new medium for reflection on culture. Rushmeier et al. elaborated on the extensive productive interactions between these fields, but the shortcoming is that no specific suggestions were made [2]. Earle indexed images for digital deployment as part of the Bloomsbury Fashion Center, which will also include the Berg Fashion Library and Fairchild Children's Book content. Earle introduced the driving factors behind buying archives, the challenges it brings, and the logic of cooperating with other possible content providers [3]. Yang et al. proposed an effective grid-based geometric restoration system for the digitization of large-scale distorted historical documents. The system can carry out geometric modeling and correction of arbitrarily deformed historical documents, as well as a visual display and manual operation of the grid to improve the accuracy of geometric restoration. Experimental results show that the proposed automatic deformation method can effectively correct arbitrarily deformed historical documents, and its performance has been improved compared with several of the latest geometric restoration methods, but the method lacks detailed design [4]. As the core part of the microcomputer, the microprocessor has more related research. Schoeberl et al. studied a processor designed for real-time systems. They designed and optimized a processor called "Patmos" for low WCET range rather than high average use case performance. Patmos is a dual-issued static scheduling RISC processor. The method cache is used as an instruction cache, and the split cache organization simplifies the WCET analysis of the data cache [5]. Through fault injection and heavy ion experiments, Filipe studied the impact of register file errors on the reliability of modern embedded microprocessors. In addition, Filipe also evaluated how different levels of compiler optimization modify the use and failure probability of processor register files, but there is no data support for this experiment [6]. Sato et al. introduced the design and experimental results of a fast singleflux quantum (RSFQ) bit serial microprocessor with small embedded random access memory (RAM) and minimum instruction set CORE e2h. And through experiments, the correct operation of all instructions was obtained, and the highspeed transmission between the instruction memory and the controller unit and between the data memory and the data path at about $50 \mathrm{GHz}$ was confirmed. However, it would be better to add a few more sets of comparative experiments to this experiment [7].

1.4. Innovation. The innovations of this article are as follows: (1) It introduces the related technologies, methods, and significance of the digital protection of folk art, and emphasizes its importance. (2) A digital folk art website was designed based on a microprocessor to achieve the purpose of recording and inheriting folk art works.

\section{Microprocessor-Based Digital Protection Method for Folk Art}

\subsection{Microprocessor under Wireless Communication}

2.1.1. Development of Wireless Communication. With the continuous development of wireless mobile communication, various communication technologies have emerged one after another. In the past ten years, wireless communication technologies have developed rapidly. The wireless mobile communication system has experienced the first generation of analog mobile phones (1G), the second generation of GSM and other digital mobile phones $(2 \mathrm{G})$, and the third generation of WCDMA, TD-SCDMA, and other digital mobile phones (3G), and the fourth-generation communication technology, LTE and IMT,-advanced these four stages. Nowadays, with the popularization of intelligent terminals and the diversification of applications, the rapid development of wireless communication has been promoted. $5 \mathrm{G}$ has entered people's vision. It has the characteristics of new multiple access, new channel coding, new modulation, and so on. The main purpose of the continuous development of wireless communication is to achieve end-to-end reliable high-speed transmission by improving bandwidth utilization and channel transmission rate [8]. In order to achieve this goal, wireless communication algorithms are constantly being developed.

With the rise of software-defined radio, the implementation of digital signal processing has evolved to a software-based form, which can bring great improvements in flexibility. The upgrade of the wireless communication system can be easily updated by a remote software download, so the use of processor arrays at the base station side has become the trend of future processing platforms. At the same time, in the terminal, due to power and volume limitations and the use of processors with high computing capabilities will also become the future trend.

2.1.2. Processor Classification. This section is classified from the architecture of the processor. The processor is mainly divided into the von Norman structure, Harvard structure, and improved Harvard structure. The von Norman structure is shown in Figure 1, which is mainly suitable for the initial processor without a pipeline structure [9].

The Harvard structure is shown in Figure 2. The instruction bus and the data bus are separated, and instructions and data can be read at the same time, which greatly improves the processing efficiency of the processor, and is mainly used in processors with higher computing speed requirements.

In the improved Harvard structure, the instruction bus and the data bus are not strictly distinguished, which facilitates the shared memory of instructions and data, improves the access speed, and is suitable for updating instructions during operation. 


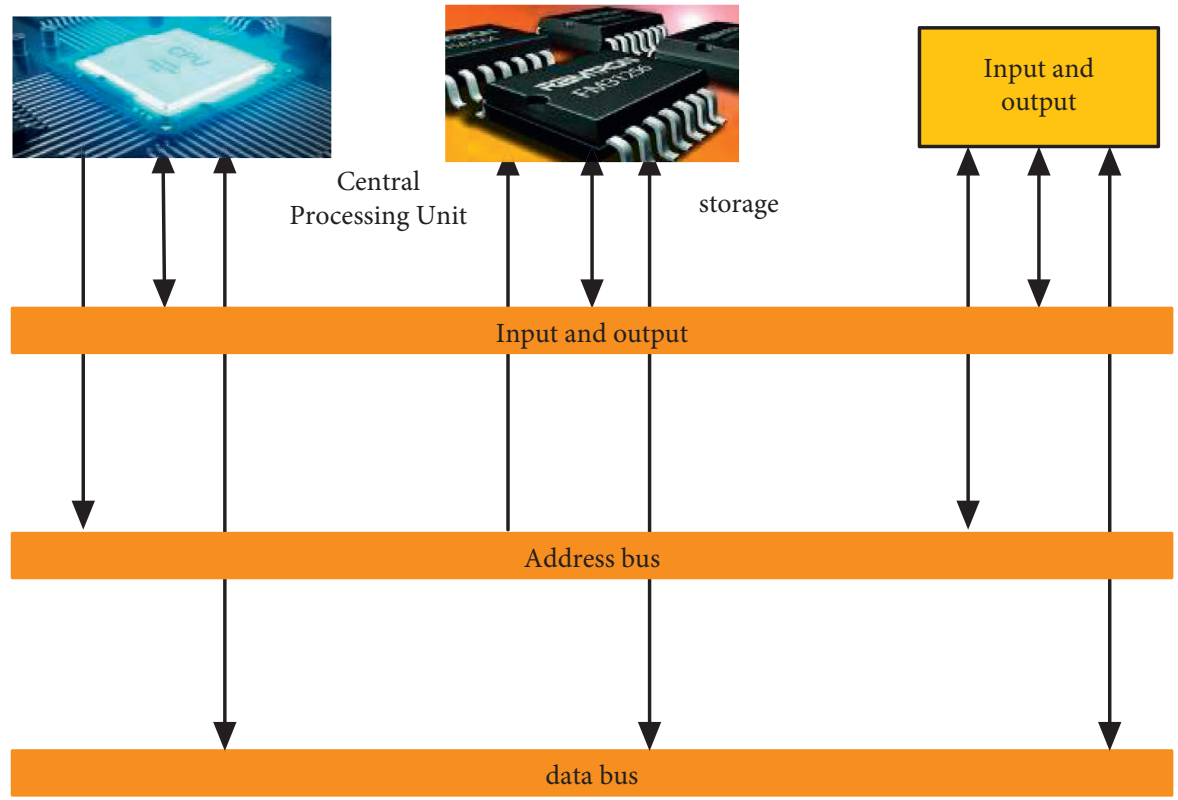

Figure 1: Von Neumann structure.

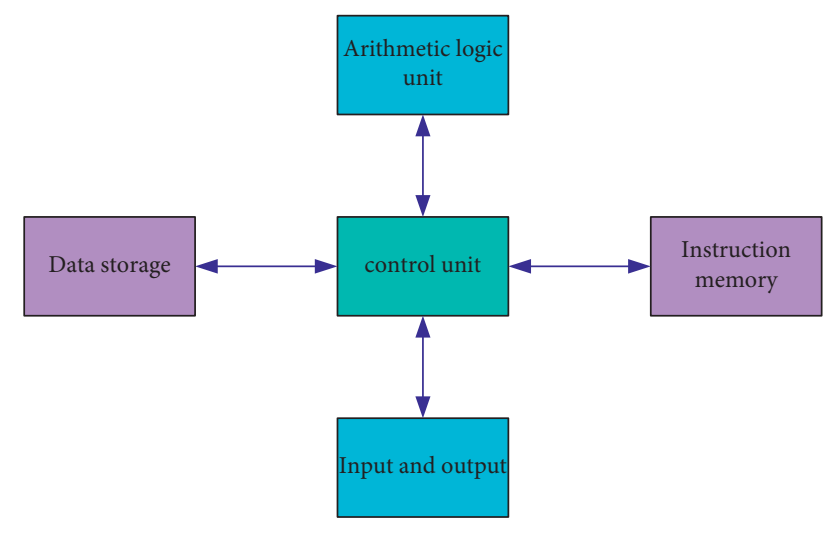

FIGURE 2: Harvard structure.

2.1.3. Processor Design. Because the processor plays an important role in all walks of life, the internal design of the processor is also a top priority. The following study will explain the internal design of the microprocessor [10].

2.1.4. Microprocessor Instruction Set Design Method. The design of the processor instruction set determines the performance of the processor. Generally, the processor instruction set is designed based on the usage of the processor. At the same time, the instruction set is also a bridge connecting high-level programming languages and processor hardware platforms, as shown in Figure 3.

In the system, algorithms with a large amount of calculations include the fast Fourier transform, impulse-limited response filter, and turbo decoding. Among them, Turbo decoding is more complicated in the current architecture design, which is not conducive to the addition of instructions, so the first two algorithms are selected for the extraction of common operations [11]. The expressions of $L$ -

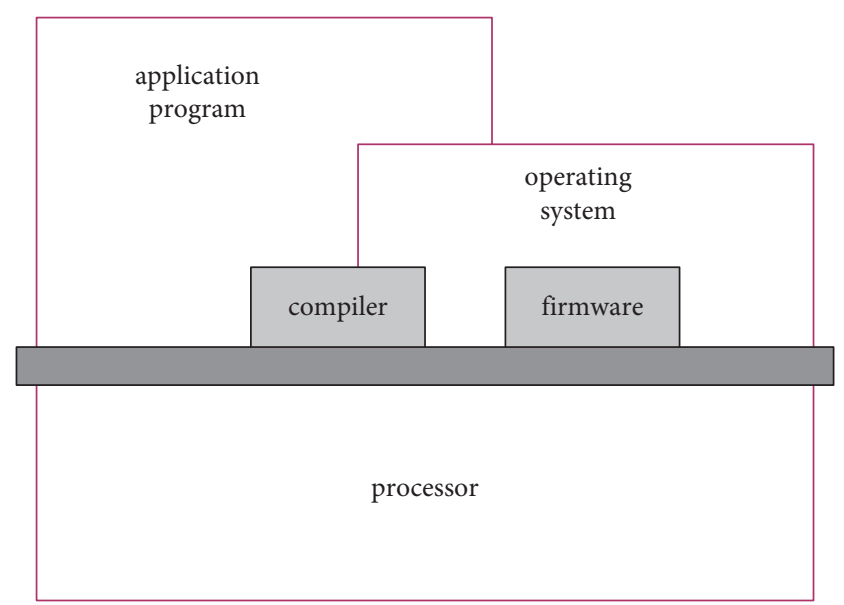

FIgURE 3: The relationship between instruction set and software and hardware.

order impulse response filtering and $N$-point fast Fourier transform are as follows:

$$
\begin{aligned}
& y(n)=\sum_{k=0}^{L} h(k) * x(n-k), \\
& y(k)=\sum_{n=0}^{N-1} X(n) * W_{N}^{k_{n}}
\end{aligned}
$$

It can be found that the multiply-accumulate operation is widely used.

2.1.5. Design of Processor Adder. The adder design used in this article is a serial method [12], as shown in Figure 4.

This section uses the carry forward adder algorithm to design the adder to avoid slow operation and delay. This adder can only obtain the carry signal $C$ of the adder by 


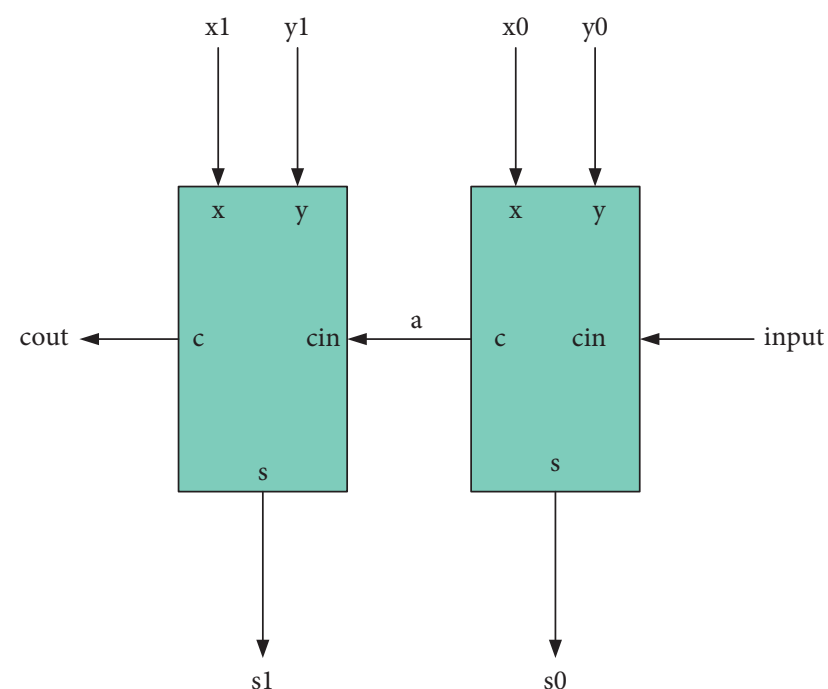

Figure 4: Adder structure.

calculating the signals $G$ and $P$. Each adder operates independently and does not interfere with each other. The calculation formula is as follows:

$$
\begin{aligned}
G_{i} & =A_{i} B_{i}, \\
P_{i} & =A_{i} \oplus B_{i}, \\
S_{i} & =A_{i} \oplus B_{i} \oplus C_{i-1}=P_{i} \oplus C_{i-1} .
\end{aligned}
$$

In the above formula, $G_{i}$ is the carry generation signal, and $P_{i}$ is the carry propagation signal [13]. It can be deduced that the $i$-th carry signal is

$$
C_{i-1}=G_{i}+P_{i} C_{i}
$$

Carrying this formula, we get

$$
C_{i}=G_{i}+P_{i} G_{i}+P_{i} P_{i-1} G_{i-1}+P_{i} P_{i-1} P_{1} P_{0} C_{0} .
$$

\subsection{Key Technologies of Fine Art Digitization}

\subsubsection{Three-Dimensional Tracking Registration Technology.} Computer vision-based three-dimensional tracking and registration technology is that the system uses the image frame containing the target object as the positioning reference in the video image sequence frame of the real scene. Through the method of feature point detection, recognition matching, and real-time tracking of the image frame of the target object, the pose relationship between the camera and the real environment can be obtained in real time. And the coordinate conversion relationship between the virtual object and the target object in the real environment is updated in real time, and then the virtual and real scene is updated in real time to complete the task of three-dimensional registration [14]. The three-dimensional tracking registration process based on computer vision mainly includes four links: image feature point detection, recognition and matching, real-time tracking of target objects, and virtual and real fusion. The main process is shown in Figure 5.
The essence of the $3 \mathrm{D}$ tracking registration process involves the conversion relationship between the world coordinate system, the camera coordinate system, the imaging plane coordinate system, and the pixel coordinate system. Through the determination of the conversion matrix between these four coordinate systems, the system can accurately superimpose the virtual object in the scene of the real environment [15]. The coordinates of any point in the threedimensional space of the real environment are set to $W\left(X_{W}\right.$, $\left.Y_{W}, Z_{W}\right)$, and then the point $X_{C}$ of the equation is $\left(X_{C}, Y_{C}\right.$, $\left.Z_{C}, 1\right)$, and the following is the conversion formula between the world coordinate system and the camera coordinate system:

$$
\left[\begin{array}{c}
X_{C} \\
Y_{C} \\
Z_{C} \\
1
\end{array}\right]=R_{W C} T_{W C}\left[\begin{array}{c}
X_{W} \\
Y_{W} \\
Z_{W} \\
1
\end{array}\right] .
$$

In this formula, $R_{W C}$ represents the relative position between the world coordinate system and the camera coordinates, and $T_{W C}$ represents the posture between the two.

The conversion of the camera coordinate system and the imaging plane coordinate system is as follows: the coordinates of the corresponding point in the imaging plane coordinate system and the camera coordinate system are defined as $(x, y)$; according to the perspective projection relationship, $x, y$ can be expressed as

$$
\begin{gathered}
x=\frac{f X_{C}}{Z_{C}}, \\
y=\frac{f Y_{c}}{Z_{C}} .
\end{gathered}
$$

Using a matrix to express the conversion relationship between the camera coordinate system and the imaging plane coordinate system is

$$
Z_{c}\left[\begin{array}{c}
x \\
y \\
1
\end{array}\right]=\left[\begin{array}{llll}
f & 0 & 0 & 0 \\
0 & f & 0 & 0 \\
0 & 0 & 1 & 0
\end{array}\right]\left[\begin{array}{c}
X_{C} \\
Y_{C} \\
Z_{C} \\
1
\end{array}\right] .
$$

In the above matrix, $f$ represents the distance between the imaging plane and the focal plane of the camera.

The transformation between the imaging plane coordinate system and the pixel coordinate system can be expressed as follows: the coordinates of the imaging point in the imaging plane coordinate system are defined as $(x, y)$, and its corresponding coordinates in the pixel coordinate system are defined as $(m, n)$, and $\left(m_{0}, n_{0}\right)$ are the coordinates in the camera image pixel coordinate system. Let $k$ be the physical size of each pixel on the $x$-axis and $y$-axis. Since the oblique distortion angle of the physical coordinates of the camera pixel is approximately 0 , the transformation matrix between the imaging plane coordinate system and the pixel coordinate system can be expressed as the following formula [16]: 


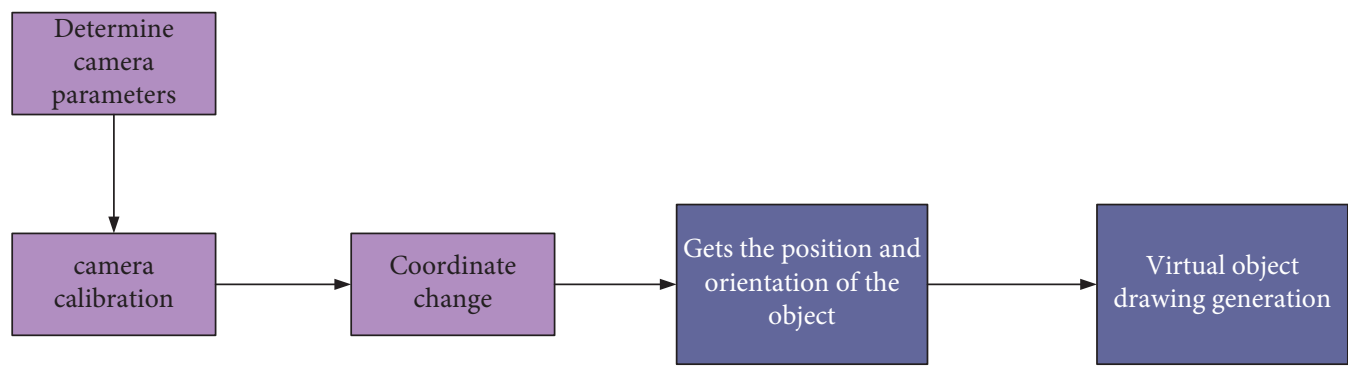

FIgURE 5: Three-dimensional tracking registration process.

$$
\left[\begin{array}{l}
m \\
n \\
1
\end{array}\right]=\left[\begin{array}{ccc}
\frac{1}{k_{x}} & 0 & m_{0} \\
0 & \frac{1}{k_{y}} & n_{0} \\
0 & 0 & 1
\end{array}\right]\left[\begin{array}{l}
x \\
y \\
1
\end{array}\right] .
$$

Supposing the scale factor of the conversion from the imaging plane coordinate system to the camera coordinate system is $\alpha=Z_{C}$. According to the previous formulas, the relationship between the pixel coordinates $(m, n, 1)$ and the three-dimensional space coordinates $(X, Y, Z, 1)$ can be obtained as follows:

$$
\begin{aligned}
\alpha\left[\begin{array}{l}
m \\
1
\end{array}\right] & =\left[\begin{array}{ccc}
\frac{1}{k_{x}} & 0 & m_{0} \\
0 & \frac{1}{k_{y}} & n_{0} \\
0 & 0 & 1
\end{array}\right]\left[\begin{array}{cccc}
f & 0 & 0 & 0 \\
0 & f & 0 & 0 \\
0 & 0 & 1 & 0
\end{array}\right] R_{W C} T_{W C}\left[\begin{array}{c}
X_{W} \\
Y_{W} \\
Z_{W} \\
1
\end{array}\right] \\
& =\lambda R_{W C} T_{W C}\left[\begin{array}{c}
X_{W} \\
Y_{W} \\
Z_{W} \\
1
\end{array}\right],
\end{aligned}
$$

where

$$
k=\left[\begin{array}{ccc}
\frac{f}{d_{x}} & 0 & m_{0} \\
0 & \frac{f}{d_{y}} & n_{0} \\
0 & 0 & 1
\end{array}\right],
$$

$K$ represents the internal parameters of the camera, including the focal length and the horizontal and vertical pixel size of the imaging plane [17].
2.2.2. Curve and Surface Modeling Theory. Free-form surface refers to the free form of spatial expansion. Generally speaking, curves and surfaces cannot be defined by linear and quadratic equations. This type of surface has a high degree of freedom, and simple basic functions cannot be used for fitting [18]. The parameter curve contains only one independent variable, and the coordinates of the points on the curve are the parameters of the curve. When the parameter is $t$, the parameter formula of the curve is as follows:

$$
\left\{\begin{array}{l}
x=x(t), \\
y=y(t), \quad t \in[a, b] . \\
z=z(t),
\end{array}\right.
$$

The point clouds scanned by the $3 \mathrm{D}$ scanner are all $3 \mathrm{D}$ coordinates. What the inverse software needs to do is to fit these point clouds into a curved surface. A curved surface is an equation with two parameters, while a curve contains only one parameter [19]. In order to understand the entire process of surface modeling, we start with the most basic quadratic uniform B-spline curve. Supposing we scan to 3 points $(0,1),(1,0)$, and $(2,0)$, according to the expression of the quadratic uniform B-spline curve:

$$
P_{i}(u)=\sum B_{j, 2}(u) V_{i+j}
$$

Among them, the basis function is

$$
\begin{aligned}
& B_{0,2}=\frac{(1-u)^{2}}{2}, \\
& B_{1,2}=\frac{\left(1+2 u-2 u^{2}\right)}{2}, \\
& B_{2,2}=\frac{u^{2}}{2} .
\end{aligned}
$$

These three formulas are the basis function expressions corresponding to different points.

We bring in the coordinates of the three vector points to calculate the parametric equation $(x, y)$ :

$$
\left\{\begin{array}{l}
x=u+\frac{1}{2} \\
y=u-u^{2}+\frac{1}{2}
\end{array}\right.
$$




\subsection{Digital Protection of Folk Art}

\subsubsection{Digital Protection Method}

(1) Digital Photography. Digital photography is the use of a digital camera to directly convert an image into a digital signal that can be recognized by a computer, and finally, an image is formed and can be output conveniently and quickly [20]. This technology does not consume any materials. If any unsatisfactory parts in the photo are found, it can operate and delete them until it is satisfied, so the work efficiency can be greatly improved. Digital photography technology is the most used method in the process of digital protection, and it is also the quickest. It can be used for standardized shooting when recording and sorting out related arts and culture, including works of art and artistic craftsmanship. It is an important way to obtain graphics and images. The size of the image file determines the speed of image transmission and affects the quality of the image. In actual use, the relationship between speed and quality should be coordinated comprehensively to avoid occupying network and hard disk resources [21].

(2) Digital Audio. Digital audio is one of the important manifestations of the digital form of folk art and is the main way to improve the performance of visual media in order to achieve the purpose of audio and picture synchronization. Loading the audio signal into the image or video can play an irreplaceable effect in other media forms. It can record the voice of the inheritor in real time or record the environmental sound to set off the theme atmosphere. Firstly, digital audio can record the sound in any environment, collect and process through recording, and then play it out by digital technical means. Secondly, digital audio technology can also create sounds and music that do not exist in reality [22]. The advantage of digital audio is that it is convenient and fast to store; basically, there is no loss of information, no distortion, and it is easy to edit. It can copy all sounds from the natural world, sample any sound, and realize and play it out in the form of digital technology.

(3) Digital Video. Digital video is mainly used for the shooting of folk art craftsmanship and the preservation of some image materials. It uses different scenes to take detailed shots of each link, combined with audio instructions to achieve the best recording method [23]. As an important digital protection technology method for folk art, digital video is mainly used to record the flow of folk crafts and the preservation of some image data. Through the use of different angles of scenes, each aspect of the craft is meticulously recorded, combined with an audio introduction to achieve the best recording mode.

(4) Three-Dimensional Panorama. Digital panorama is a virtual reality technology that uses digital methods to display 360-degree panoramas. The specific production method is to use digital photography or video to take a 360-degree circle shot of the real object to form one or several groups of photos or videos and then collage them into panoramic images. Through three-dimensional tools and technology, the real scene and virtual modeling are integrated, and then the obtained data are built in the real-life environment. With the atmosphere of audio, lighting, and models, people can witness the occurrence of historical events, experience the grandeur of historical monuments in real life, and get the combination of virtual modeling and real-time shooting, as well as realistic interaction effects, which makes people feel immersive [24].

2.3.2. The Value of Digitalization for the Protection of Folk Art. With the improvement of the level of science and technology, the rapid development of digital information collection, processing, and inheritance has greatly promoted the progress of modern civilization and innovated the way of social civilization and the protection of traditional folk art. Computer technology has begun to be fully applied to the protection and development of folk art resources. various folk art information are available through mobile phones, where archiving, development, and research have an operating specification and implementation standard, which greatly support the protection and application research of folk art. Folk art is not an exquisite art form but is constantly changing and developing. The corresponding digital acquisition and collection of it are more complex and diverse than the operation of traditional static cultural heritage [25]. Using radio, television, Internet, and other communication facilities, under relevant regulations and standards, can not only realize the widespread dissemination of folk culture but also make it possible to show art works to people in a more direct and intrusive way in the virtual world built by computer technology. Figure 6 shows the integration and application of modern new technologies.

It can be seen from the figure that the technologies for folk art protection include digital technology, modeling technology, resource management technology, and visualization technology. The integration of these technologies can establish an effective folk art protection mechanism.

\section{Microprocessor-Based Folk Art Digital Protection Website Design Experiment}

This experiment is based on the microprocessor design of a digital protection of folk art website, and the website is designed on the basis of the microprocessor design. So, this experiment is mainly divided into two parts, namely, the design part of the microprocessor and the design part of the art digital website.

\subsection{The Design Part of the Microprocessor}

3.1.1. System Software Process. After the software is turned on, the system enters the initialization phase. This includes the initialization of the microcontroller, the initialization of the memory controller, and the initialization of the analogdigital converter. The modes represented by the mode control pins are shown in Table 1. 


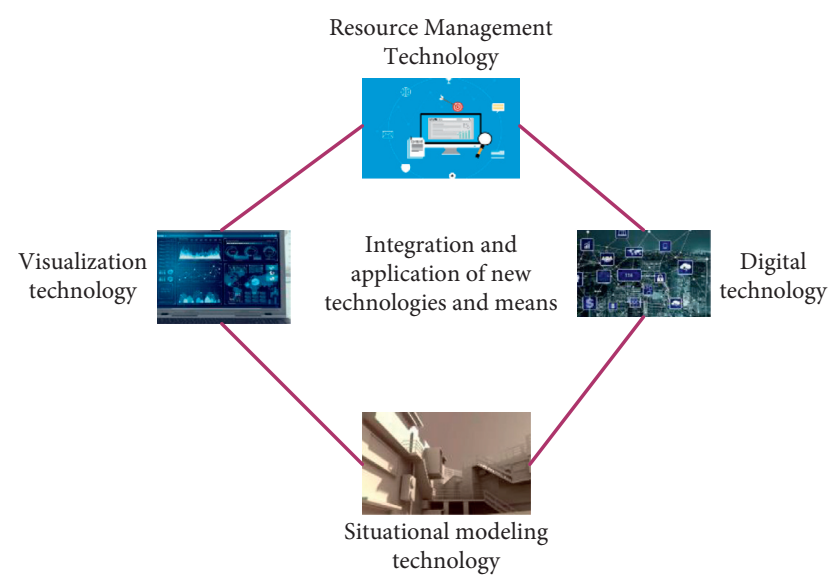

FIgURe 6: Converged application of new technologies.

TABle 1: Pin mode table.

\begin{tabular}{lc}
\hline Mode pin value & Selection of mode \\
\hline 00 & Missile borne mode I \\
01 & Missile borne mode II \\
$1 \mathrm{X}$ & Ground mode \\
\hline
\end{tabular}

Table 1 shows the sampling parameters in different modes.

3.1.2. Control Program Debugging. Memory control mainly includes memory block erasing, page programming, page reading, chip reset, and read chip ID operations to facilitate hardware debugging. The control command words are shown in Table 2. The command, address, and data ports of the chip are multiplexed. The memory chip distinguishes whether the input data is a command word, an address word, or data which is mainly determined by the command and address latch pins.

3.2. The Design Part of the Fine Art Digital Website. Drawing on the mature experience and methods of how to protect and develop other folk art resources, the art digital system needs to store and record relevant information about art resources. With this as a starting point, a database with folk art works as the primary data was established. On this basis, developing an Internet display and communication platform realizes further integration and development of resources through the website and establishes a folk art website.

\subsubsection{The Overall Construction of the Digital Art Website.} Using the database of folk art works is to establish a website publicity platform to effectively protect and publicize works. Based on this, although folk crafts cannot be displayed systematically and comprehensively, more folk crafts resources can be absorbed on this basis so that the folk crafts database can be expanded and strengthened. If the website is relatively mature, it can comprehensively introduce and promote local folk crafts and further enhance the protection and inheritance mechanism of folk crafts; its user function diagram is shown in Figure 7.

3.2.2. Site-wide Search System. The website provides the function of searching folk art works throughout the website. The website retrieval system supports various types of information retrieval and provides the retrieval function of the main information on the website. The method of saving system information is the static HUML (for static web pages, if the function of the website is relatively simple and the amount of content updates is not large, it will be easier to use pure static web pages) of the file system and the information read into the database, so the system uses two search engines. One is the full-text search function of the file system, and the other is the search function of the database system. The main table of the work database is shown in Table 3.

3.2.3. Picture Information Display System. The whole system can be divided into several parts: one is the first picture display; in this section, users can see the online catalog of folk art works and see all the art images. If the website is updated, viewers can get the latest product catalog. The second is to update and add new image information. The website staff does not rely on web production companies and programmers, and can fully control, edit, or supplement the website at any time. The third is a detailed image introduction. The website will provide a comprehensive description of the image information. The audience can fully understand all the images so that they can flexibly choose to download and submit their own opinions to the images on the message board or online questionnaire. The fourth is the search engine function; users can quickly and accurately find the image. As a result, users can quickly find the images they want, saving unnecessary time.

3.2.4. Digital Protection Design of Fine Art Works. The system uses the shared graphic characteristics and color matching styles of the recorded patterns to generate designs through analysis and comparison. It is possible to judge and modify the design works, and also to process the data related to graphics, such as editing, zooming in, zooming out, translation, and rotation, to establish a computer-aided design database. On the one hand, it can use the method of establishing a pattern database to store folk art works; on the other hand, it can use the computer to realize the recreation of patterns. The digitization of art works is mainly to provide theory and paradigm for the digital mining, preservation, dissemination, and development of folk art, and to provide methods and basis for the protection of folk art in the future and the inheritance of education.

\subsection{Experimental Results and Analysis}

3.3.1. Verification of the Storage Function of the Microprocessor. When verifying the storage function, the wireless transceiver module is connected to the acquisition board, and another identical acquisition board B is placed on 
TABLE 2: Memory chip command word.

\begin{tabular}{lccc}
\hline Command name & Command word 1 & Number of valid address cycles & Command word 2 \\
\hline Reset & $0 * F F$ & 0 & - \\
Read chip status & $0 * 20$ & 0 & - \\
Page read & $0 * 00$ & 5 & $0 * 30$ \\
Page storage & $0 * 80$ & 5 & $0 * 10$ \\
Read chip ID & $0 * 90$ & 1 & - \\
\hline
\end{tabular}

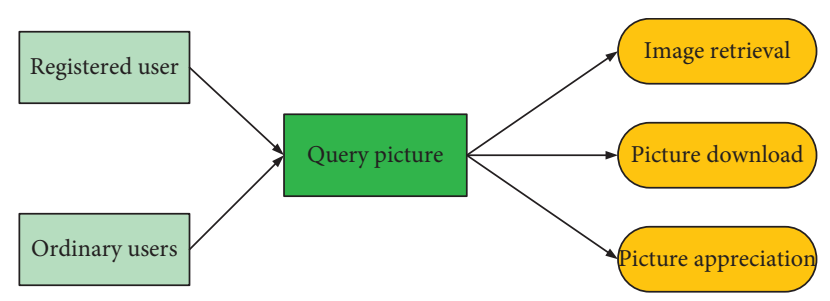

FIgURE 7: User function diagram.

TABLE 3: Database table.

\begin{tabular}{lcc}
\hline Work ID & Auto number & Long integer \\
\hline Name & Text & 50 \\
Style & Text & 50 \\
Category & Text & 50 \\
Age information & Text & 50 \\
Entry time & Time type & Automatic acquisition \\
Large picture of the work & Picture & Database \\
\hline
\end{tabular}

the bracket where the fuze body is placed, and the acquisition board B is used to collect and send signals. It is received and stored by the acquisition board A on the test bench; the sampling rate is $40 \mathrm{KHz}$, and the acquisition time is $40 \mathrm{~s}$. The acquisition is also performed under the interference of the sweep frequency and amplitude modulation signal and the sweep frequency and amplitude signal. The signal comparison under sweep frequency and amplitude modulation interference is shown in Figure 8. The left picture of Figure 8 is the signal received by the acquisition board, and the right picture is the signal collected by the sound card. It can be seen that the signal is slightly messy after $30 \mathrm{~s}$, and the accuracy is basically maintained for the rest of the time.

In the case of frequency sweep interference and breakpoint interference, the signal comparison diagram is shown in Figure 9.

Judging from the obvious large signal, the two acquisition modes are basically the same, but they are slightly different in the delicacy of the entire signal. The two acquisition modes maintained the same level at the time of the interception and basically restored the real signal, which shows that the sampling rate fully meets the requirements of the signal. In summary, this design can meet the needs of the project and achieve the expected results.

3.3.2. Satisfaction Survey on Art Digital Website. In the art digital system based on the above design, this experiment designed a simple satisfaction survey questionnaire on the website. Let the user rate be the satisfaction of the website.
This questionnaire first counts the user's age and then allows the user to rate the score. The score is averaged, and the user's age and scoring results are obtained, as shown in Figure 10.

It can be seen from Figure 10 that the most users of this website are young people aged 18-29, which proves that the preservation and dissemination of folk art works in the form of a website are more favored by young people. The second place is people in the 30-39 age group, further confirming that the website model is more attractive to young people. In the scoring module, the group with the highest average score is the group older than 59 years old, and the average score is 90 points. For $18-29$, the group with the most users, the average score is only 77 points, which proves that the website has great improvements in viewing and user experience.

\section{Discussion}

Abundant works of fine arts are the precious spiritual wealth of the country. It is the responsibility of people to let friends all over the world and future generations enjoy this cultural blessing, feel its powerful charm, and pass it on. It is people's responsibility to preserve these works carefully, protect them carefully, and make them widely popular. This is also very important for promoting traditional culture and improving national cultural competitiveness. Digital technology provides a reliable technical foundation for achieving this goal. Using digital technology, let folk crafts be recorded in digital form and stored in the computer to achieve the purpose of permanent preservation, protection, and use. Digital folk art should also use new information dissemination and 

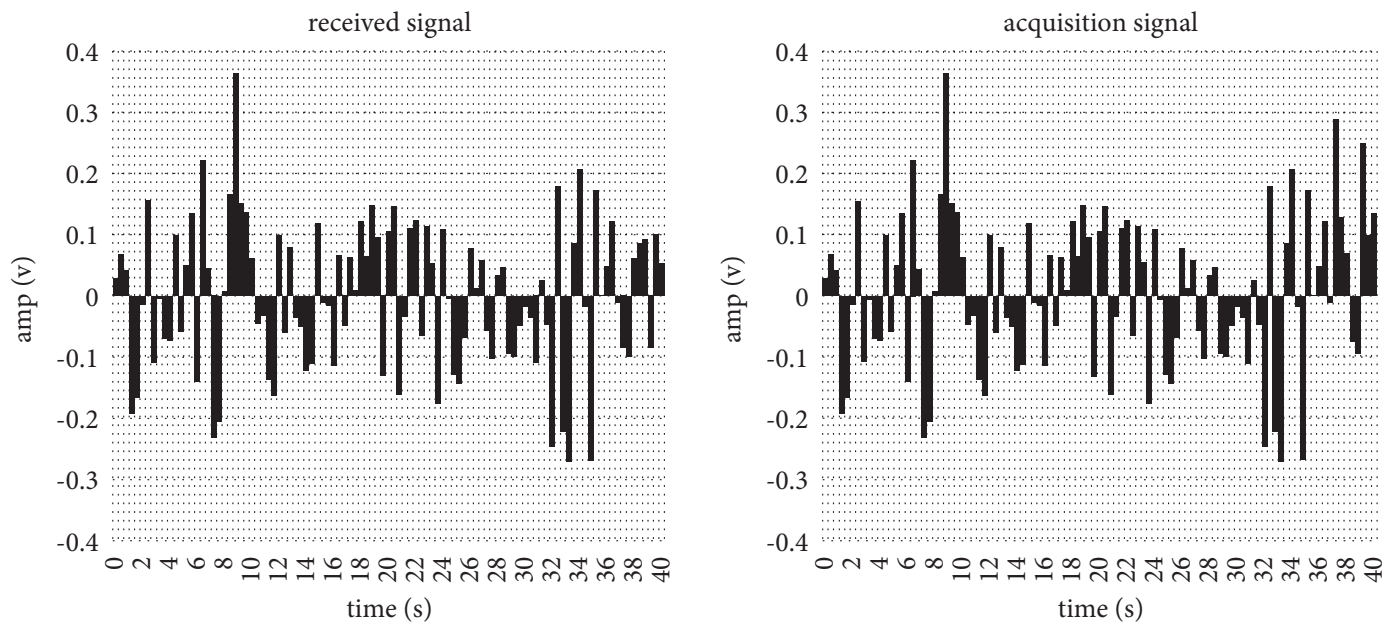

FIgURE 8: Signal comparison chart under sweep frequency and amplitude modulation interference.
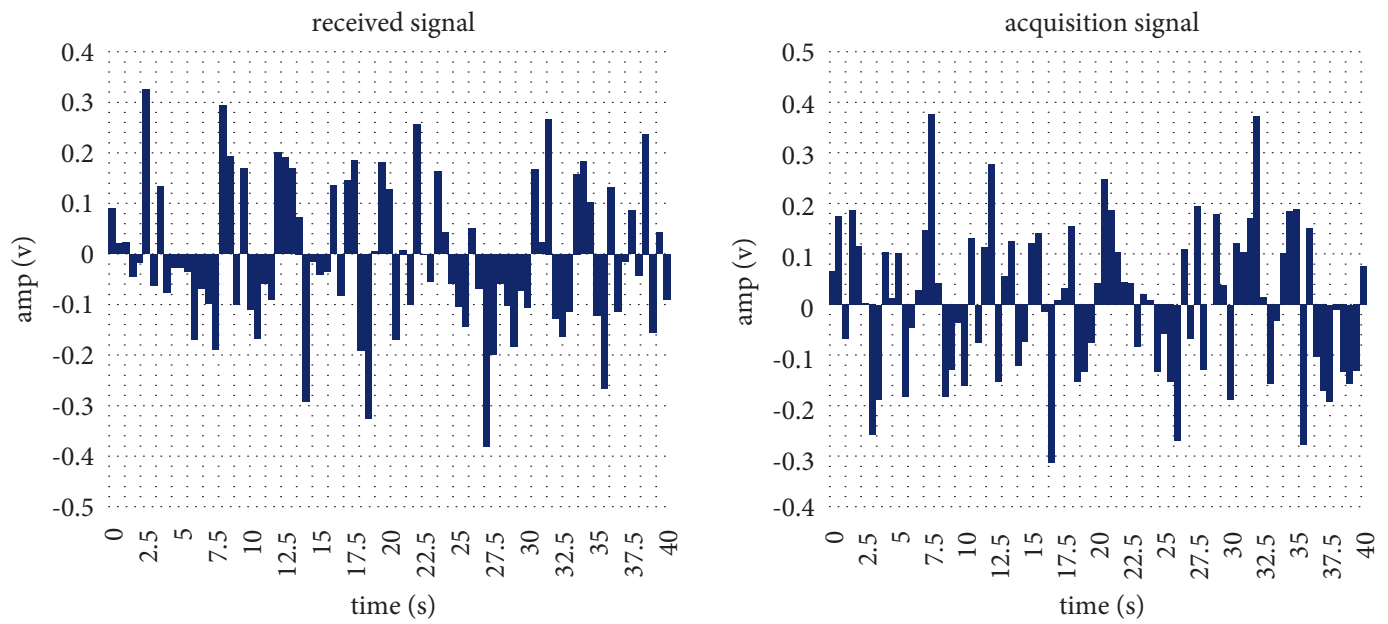

FiguRE 9: Signal comparison chart under sweep frequency sweep interference.
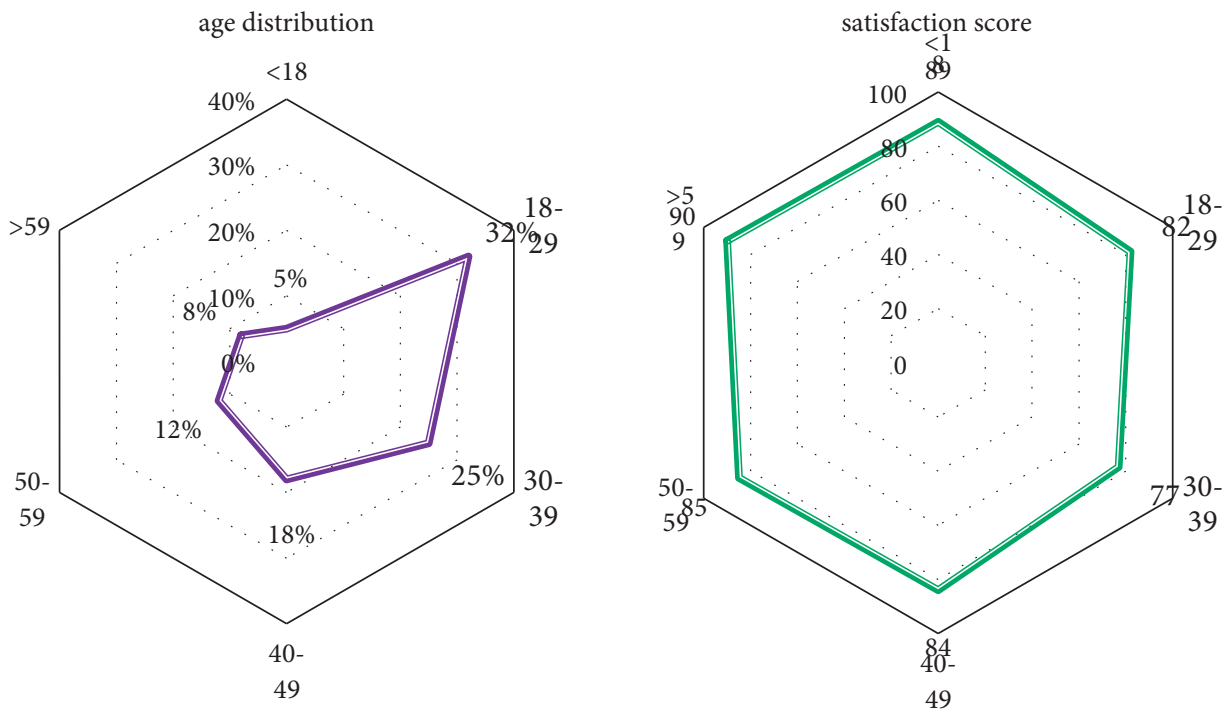

Figure 10: User age and satisfaction statistics. 
inheritance platforms, especially mobile media to exert its influence, and lay a solid foundation for the development of folk art.

\section{Conclusion}

This article introduces microprocessors and related methods of art digitalization, explains the digital protection methods of art, and also explains its significance. In the end, this paper designs a microprocessor-based digital art protection website and designs the microprocessor and the website, respectively, aiming to preserve folk art works through the website, pass it on, and spread it widely. The experiment in this article not only tested the performance of the microprocessor but also investigated the satisfaction degree of the website; the result is obtained as follows: (1) The sampling rate of the microprocessor fully meets the requirements of the signal, which proves that the design of the microprocessor can meet the needs of the project and achieve the expected results. (2) Among the users of digital folk art websites, the most are young people aged 18-29, accounting for $32 \%$. (3) Those who have the highest average score on the website are those over 59 years old, with an average score of 90 points. In summary, it can be seen that the website is more attractive to young people, but the satisfaction of the elderly is higher.

\section{Data Availability}

The data used to support the findings of this study are available from the author upon reasonable request.

\section{Conflicts of Interest}

The author declares no conflicts of interest.

\section{Acknowledgments}

This work was supported by Hunan social science fund project "Research on the Coupling of Art and Digital Media Creative Industry of Western Hunan minorities" (Project number: 17YBX006).

\section{References}

[1] J. E. Burns, "The aura of materiality: digital surrogacy and the preservation of photographic archives," Art Documentation: Journal of the Art Libraries Society of North America, vol. 36, no. 1, pp. 1-8, 2017.

[2] H. Rushmeier, F. Samsel, and J. Zhang, "Art and cultural heritage," IEEE Computer Graphics and Applications, vol. 40, no. 3, pp. 17-18, 2020.

[3] K. Earle, "From runway to platform: the creation of a digital fashion photography archive," Art Libraries Journal, vol. 42, no. 01, pp. 47-54, 2017.

[4] P. Yang, A. Antonacopoulos, C. Clausner, S. Pletschacher, and J. Qi, "Effective geometric restoration of distorted historical document for large-scale digitisation," IET Image Processing, vol. 11, no. 10, pp. 841-853, 2017.
[5] M. Schoeberl, W. Puffitsch, S. Hepp, B. Huber, and D. Prokesch, "Patmos: a time-predictable microprocessor," Real-Time Systems, vol. 54, no. 2, pp. 389-423, 2018.

[6] F. M. Lins, L. A. Tambara, F. L. Kastensmidt, and P. Rech, "Register file criticality and compiler optimization effects on embedded microprocessor reliability," IEEE Transactions on Nuclear Science, vol. 64, no. 8, pp. 2179-2187, 2017.

[7] R. Sato, Y. Hatanaka, Y. Ando et al., "High-speed operation of random-access-memory-embedded microprocessor with minimal instruction set architecture based on rapid singleflux-quantum logic," IEEE Transactions on Applied Superconductivity, vol. 27, no. 4, pp. 1-5, 2017.

[8] M. Demler, "IBM trains in analog to save AI power," Microprocessor Report, vol. 32, no. 8, pp. 23-26, 2018.

[9] F. Faggin, "How we made the microprocessor," Nature Electronics, vol. 1, no. 1, p. 88, 2018.

[10] K. Korsah, "Application of microprocessor-based equipment in nuclear power plants-technical basis for a qualification methodology," Office of Scientific \& Technical Information Technical Reports, vol. 4, no. 4, pp. 206-207, 2018.

[11] T. Lowe, "Artists' books and the problem of digital preservation," Preservation, Digital Technology \& Culture, vol. 46, no. 4, pp. 132-135, 2017.

[12] S. Balietti, "The human quest for discovering mathematical beauty in the arts," Proceedings of the National Academy of Sciences, vol. 117, no. 44, pp. 27073-27075, 2020.

[13] D. R. Seshadri, C. Drummond, J. Craker, J. R. Rowbottom, and J. E. Voos, "Wearable devices for sports: new integrated technologies allow coaches, physicians, and trainers to better understand the physical demands of athletes in real time," IEEE Pulse, vol. 8, no. 1, pp. 38-43, 2017.

[14] T. P. Nguyen, T. T. Tran, and D. N. Vo, "Improved stochastic fractal search algorithm with chaos for optimal determination of location, size, and quantity of distributed generators in distribution systems," Neural Computing and Applications, vol. 31, no. 11, pp. 7707-7732, 2019.

[15] J. Vissers, F. Van den Bosch, A. Bogaerts et al., "Scientific user requirements for a herbarium data portal," Phyto Keys, vol. 78, no. 78, pp. 37-57, 2017.

[16] A. Oram, "Constricting an art form, digitization can open it as well," Communications of the ACM, vol. 60, no. 6, p. 9, 2017.

[17] L. Gwennap, "FPGAs accelerate deep learning," Microprocessor Report, vol. 31, no. 11, pp. 22-24, 2017.

[18] E. C. Prinsen, M. J. Nederhand, H. S. Sveinsdóttir et al., "The influence of a user-adaptive prosthetic knee across varying walking speeds: a randomized cross-over trial," Gait \& Posture, vol. 51, no. Complete, pp. 254-260, 2017.

[19] T. Liu, C. C. Chen, and L. Milor, "Comprehensive reliabilityaware statistical timing analysis using a unified gate-delay model for microprocessors," IEEE Transactions on Emerging Topics in Computing, vol. 6, no. 99, pp. 219-232, 2018.

[20] L. Wright and L. Ayers, "Mitigation of undesired operation of recloser controls due to distribution line inrush," IEEE Transactions on Industry Applications, vol. 53, no. 1, pp. 8087, 2017.

[21] E. Berman, J. Bound, and S. Machin, "Implications of skillbiased technological change: international evidence," Papers, vol. 113, no. 4, pp. 1245-1279, 2018.

[22] C. Andreazzi, V. Rademaker, R. Gentile, H. Herrera, A. Jansen, and P. D'Andrea, "Wireless self-powered visual and NDE robotic inspection system for live gas distribution mains," The Zoologist, vol. 28, no. 3320, pp. 349-350, 2017.

[23] K. Mcguire, G. De Croon, C. De Wagter, K. Tuyls, and H. Kappen, "Efficient optical flow and stereo vision for 
velocity estimation and obstacle avoidance on an autonomous pocket drone," IEEE Robotics and Automation Letters, vol. 2, no. 2, pp. 1070-1076, 2017.

[24] S. Lavington, "An appreciation of Dina st Johnston (1930-2007) founder of the UK's first software house," Computer Journal, vol. 52, no. 3, pp. 378-387, 2018.

[25] D. Chen, J. Cong, S. Gurumani, W. M. Hwu, K. Rupnow, and Z. Zhang, "Platform choices and design demands for IoT platforms: cost, power, and performance tradeoffs," IET Cyber-Physical Systems: Theory \& Applications, vol. 1, no. 1, pp. 70-77, 2017. 